\title{
Thermal nucleation of the normal state in superheated superconducting tin grains
}

\author{
H. Dubos \\ Groupe de Physique des Solides (UA 17 CNRS), Universités Denis Diderot and Pierre et Marie Curie, 75251 Cedex 05, Paris, France \\ and Centro de Física Nuclear, Universidade de Lisboa, 1699 Lisbon, Portugal \\ T. A. Girard \\ Centro de Física Nuclear, Universidade de Lisboa, 1699 Lisbon, Portugal \\ and Groupe de Physique des Solides (UA 17 CNRS), Universités Denis Diderot and Pierre et Marie Curie, 75251 Cedex 05, \\ Paris, France \\ G. Waysand \\ Groupe de Physique des Solides (UA 17 CNRS), Universités Denis Diderot and Pierre et Marie Curie, 75251 Cedex 05, Paris, France \\ P. Perrier and V. Jeudy \\ Groupe de Physique des Solides (UA 17 CNRS), Universités Denis Diderot and Pierre et Marie Curie, 75251 Cedex 05, Paris, France \\ and Centro de Física Nuclear, Universidade de Lisboa, 1699 Lisbon, Portugal \\ D. Limagne \\ Groupe de Physique des Solides (UA 17 CNRS), Universités Denis Diderot and Pierre et Marie Curie, 75251 Cedex 05, Paris, France \\ J. Seco \\ Centro de Física Nuclear, Universidade de Lisboa, 1699 Lisbon, Portugal \\ J. I. Collar \\ PPE Division/CERN, 1211 Geneva 23, Switzerland; \\ Groupe de Physique des Solides (UA 17 CNRS), Universités Denis Diderot and Pierre et Marie Curie, 75251 Cedex 05, Paris, France; \\ and Centro de Física Nuclear, Universidade de Lisboa, 1699 Lisbon, Portugal \\ (Received 5 March 1998)
}

\begin{abstract}
Suspensions of superheated type-I superconducting Sn microspheres of 16-25 and 10-25 $\mu \mathrm{m}$ diameters were irradiated at temperatures below $1 \mathrm{~K}$ and above $2 \mathrm{~K}$ by decay electrons from ${ }^{14} \mathrm{C}$ and ${ }^{35} \mathrm{~S}$, respectively. Measurements of the radiation-induced gaps in the superheating transition curves indicate a full heating of the smallest grain volume of each suspension, contrary to a partial heating model based on the difference between quasiparticle relaxation and diffusion rates in $\mathrm{Sn}$. The phonons are in thermal equilibrium with the quasiparticles when the normal state is nucleated. [S0163-1829(98)05834-2]
\end{abstract}

\section{INTRODUCTION}

The thermodynamic response of metastable type-I superconducting microspheres to irradiation has received considerable attention in recent years as a result of their possible applications in particle detection. ${ }^{1,2}$ Thermal nucleation of the normal state in the superheated material is induced by the interaction of radiation if the energy deposited is sufficient to raise its temperature across the phase boundary. How much of the volume is required to be heated in order to induce nucleation of the normal state has, however, been a point of some dispute.

There are essentially two possibilities: some or all. In "local-heating" descriptions, only a fraction of the volume is required; in a "global-heating" description, the entire volume is uniformly heated above the phase line before the normal state is nucleated.

To our knowledge, a complete description of "local heating" has never been satisfactorily elaborated. Generally, the volume fraction has been related to the heating of an equa- torial surface area, or nucleation germ (surface defect, boundary, or impurity). The significance of defects was demonstrated by single-microsphere (hereafter referred to as "grain") experiments in which a variation of the superheated-to-normal transition field was observed for variation of the grain orientation relative to the applied field. ${ }^{2,3}$ Similar results were obtained on single-grain rotations under irradiation, ${ }^{3}$ the effect is more pronounced at increasingly lower temperatures.

On the basis of a series of irradiation measurements with single grains, Frank et al. ${ }^{4}$ recently advanced a "unified" heating model that combines both "local" and "global" heating behaviors by whether or not an equatorial defect is heated above the phase line before or with the uniform heating of the grain, which in turn depends on (1) the location of the interaction site relative to that of the defect, and (2) the difference between the materials-dependent thermal relaxation and diffusion rates of the excited quasiparticles. For materials in which the diffusion time is significantly less than the phonon relaxation time, no local temperature can be es- 


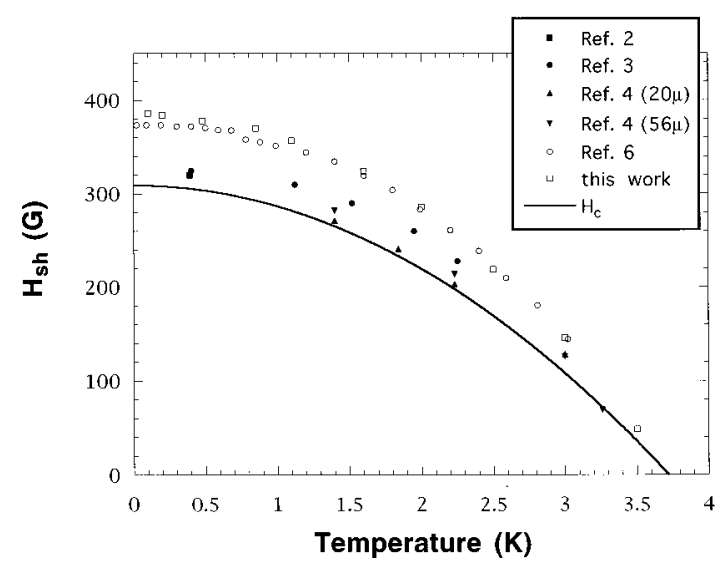

FIG. 1. Compilation of recent experimental measurements of $H_{\text {sh }}(T)$ in Sn microspheres. The solid line represents the contour of the thermodynamic critical field, obtained from Ref. 8.

tablished: the defect is uniformly heated with the grain volume, resulting in a global-heating behavior. In contrast, for materials in which the relaxation is sufficiently faster than the diffusion, such as $\mathrm{Sn} / \mathrm{In}$, only a "warm spot" is created, which if sufficiently near a defect provokes its nucleation before the remainder of the volume, resulting in a localheating behavior.

The model of Ref. 4 comes closest to including the detailed microscopic aspects of the involved energy transport. As appealing as it may appear, and independent of questions regarding the applicability of the near-equilibrium relaxation rates of Kaplan et al. ${ }^{5}$ in such nonequilibrium situations, the experimental basis given for the model must, however, be discounted in toto: neither the Sn grains of Refs. 3 and 4 nor the In grains of Ref. 4 exhibited the full superheating field $\left(H_{\mathrm{sh}}\right)$. A partial compilation ${ }^{3,4,6,7}$ of recent $H_{\text {sh }}$ measurements for Sn is shown in Fig. 1. The fact that the transition fields of Refs. 3 and 4 are near the bulk thermodynamic critical field ${ }^{8}\left(H_{c}\right)$ suggests that the observed transitions occurred from intermediate states, ${ }^{9}$ which would both lower the transition fields and generate the low-energy tail in the irradiation-induced transition spectra that are observed.

Nonetheless, the possibility of any local-heating behavior for Sn has a significant impact on the current development of Sn-based devices for particle-detection applications, since only those materials exhibiting a global-heating behavior can generate a well-defined energy threshold. The experiments of Ref. 4 were performed on relatively large (34-46 $\mu \mathrm{m})$ diameter grains using heavily ionizing $4-\mathrm{MeV} \alpha$ particles that are generally stopped within about $10 \mu \mathrm{m}$ of the grain surface. We here report a series of experiments on suspensions of smaller diameter, fully metastable $\mathrm{Sn}$ grains, under irradiation by the decay electrons of ${ }^{14} \mathrm{C}$ and ${ }^{35} \mathrm{~S}$ in which the energy depositions are distributed across the grain volumes, which clearly demonstrate a global heating in their normalstate nucleation. Although single-grain measurements in principle yield more precise results, uncertainties arising from size distributions and local-field effects can be reduced by working with well-separated suspensions of grains. This approach does not require the extreme sensitivity necessary in single-grain measurements, and has generally yielded more accurate and reliable determinations of the GinzburgLandau parameter in type-I superconductors. ${ }^{2,7}$ In Sec. II, we

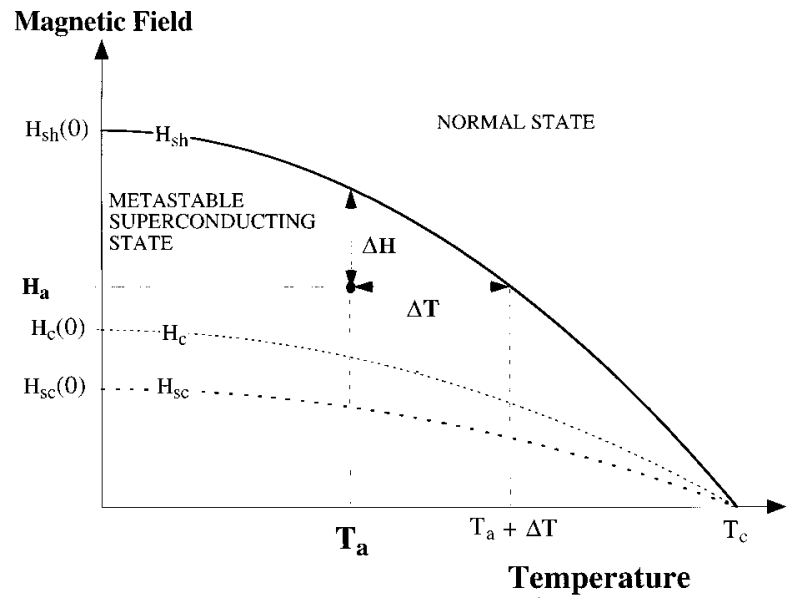

FIG. 2. A phase diagram for a single microsphere; $H_{l}(T)$ $=(3 / 2) H_{a}$.

describe in further detail the distinction between global- and local-heating models, indicating their different manifestations in single-grain and multigrain experiments. Section III describes our experiments, the results of which are given in Sec. IV.

\section{THEORETICAL CONSIDERATIONS}

\section{A. Single grain}

The phase diagram of a single superheated grain is shown in Fig. 2. The precise location of a grain within the diagram is ideally at $\frac{3}{2}$ the applied field $\left(H_{a}\right)$, corresponding to its maximum local surface field as a result of demagnetization. The presence of a nucleation germ effectively raises this position nearer the phase line as a result of its ability to scatter quasiparticles. $^{10}$

The temperature distance $(\Delta T)$ to the phase boundary is fixed by the grain location $\left(T_{a}, H_{a}\right)$. Thermal nucleation of the normal state depends only on the magnitude of the deposited energy $(\Delta E)$ :

$$
\Delta E \leqslant V_{\text {heated }} \int_{T_{a}}^{T_{a}+\Delta T} C_{s} d T,
$$

where $V_{\text {heated }}$ is the heated volume of the grain; $C_{s}$ is the volume specific heat of the superconductor, given by

$$
C_{s}=\left[a \gamma T_{c} e^{\left.-b T_{c} / T_{a}+\alpha T_{a}^{3}\right]},\right.
$$

where $\{a, b, \alpha, \gamma\}$ are materials-dependent parameters. The first and second terms of Eq. (2) describe the electron and phonon contributions, respectively. In Fig. $2, T_{a}+\Delta T$ is related to the superheated field by

$$
T_{a}+\Delta T=\left[T_{a}^{2}+\frac{3}{2} T_{c}^{2}\left\{\Delta H / H_{\mathrm{sh}}(0)\right\}\right]^{1 / 2},
$$

which is derived from the BCS temperature variation of the supercritical field, ${ }^{11}$

$$
H_{\mathrm{sh}}^{\mathrm{local}}(T)=H_{\mathrm{sh}}^{\mathrm{local}}(0)\left[1-\left(T_{a} / T_{c}\right)^{2}\right]
$$

The lowest $H_{a}$ at which a grain transition occurs, corresponding to the largest induced $\Delta T$, is given by 


$$
\int_{T_{a}}^{T_{a}+\Delta T_{\max }} C_{s} d T=\Delta E_{\max } / V_{\min },
$$

where $\Delta E_{\max }$ is the maximum energy deposition in the grain, and $V_{\text {min }}$ is the minimum $V_{\text {heated }}$. For a sufficiently large energy of the incident electron, $\Delta E$ in a grain is approximately linear with the electron range. This implies that $\Delta E_{\max }$ is proportional to the grain radius $(R)$. In a globalheating model, the grain is uniformly heated, such that $V_{\text {min }} \equiv V_{\text {grain }}, \quad$ and $\quad \Delta E_{\text {max }} / V_{\text {grain }}=\int_{T_{a}}^{T_{a}+\Delta T_{\max }} C_{s} d T \approx R^{-2}$ $=$ const, independent of temperature.

In local-heating descriptions, nucleation occurs when the requisite temperature increase reaches a defect near the grain equator. For energy depositions near this defect, the temperature increase reaches a maximum above the equilibrium value before the full grain volume; in the extreme case, $V_{\min }$ corresponds to the defect, and the required depression of the order parameter implies ${ }^{10}$ a scaling with the coherence length $(\xi)$. Since $\xi(T) \approx \xi_{0}\left(1-T / T_{c}\right)^{-1 / 2},{ }^{12}$

$$
\Delta E_{\max } / V(\xi) \approx\left[3 \Delta E_{\max } / 4 \pi \xi_{0}^{3}\right]\left[1-T / T_{c}\right]^{1.5},
$$

where $\xi_{0}$ is the Pippard coherence length of the pure metal (Sn: $0.2 \mu \mathrm{m}$ ). For energy depositions further away from the defect site, $V_{\text {heated }} \approx 0.5 V_{\text {grain }}+V_{\text {defect }} \approx 0.5 V_{\text {grain }} .{ }^{13}$ In any event, the transition occurs at a lower $H_{a}$ than expected from $\Delta E_{\text {max }} / V_{\text {grain }}$.

In the model of Ref. 4, the local-heating behavior of $\mathrm{Sn}$ results from the short time scale $(\approx 1 \mathrm{~ns})$ for quasiparticle relaxation to the energy gap by phonon emission, relative to the longer spreading time $(\approx 10 \mathrm{~ns})$ of the quasiparticles across the grain diameter. In this case, $V_{\text {heated }} \leqslant V_{\text {grain }}$ : the quasiparticles quickly relax to phonons, creating a "warm spot" within the grain volume which then spreads diffusively. Although the warm quasiparticles continue to exchange energy with cold phonons during the diffusion process, the normal-state nucleation in these materials is initiated prior to a full warming of the phonon system; for the Sn results of Ref. 4, only 20-50\% of the deposited energy is in the phonon system.

\section{B. Multigrains}

The situation for an ensemble of grains is a priori more complicated than for a single grain, owing to the inherent variation of the individual local fields resulting from the distribution of sizes, defects, and diamagnetic interactions between the grains. ${ }^{14}$ These yield an apparent spreading of the transition fields of the ensemble, as shown in the typical unirradiated experimental differential superheating curve of a test suspension of Fig. 3, which was recorded during a ramp of $H_{a}$ from zero to well above $H_{\mathrm{sh}}$ at fixed temperature. The width of the curve results from the local-field variations, and for small filling factors corresponds to the distribution of local magnetic-field states populated by the grains. The last grains to undergo a transition are those more metallurgically perfect, and they experience virtually no diamagnetic effects since the majority of the previously superconducting ensemble has already become normal.

Reduction of the filling factor reduces the effects of the diamagnetic interactions. ${ }^{15}$ Nonetheless, given the nonuni-

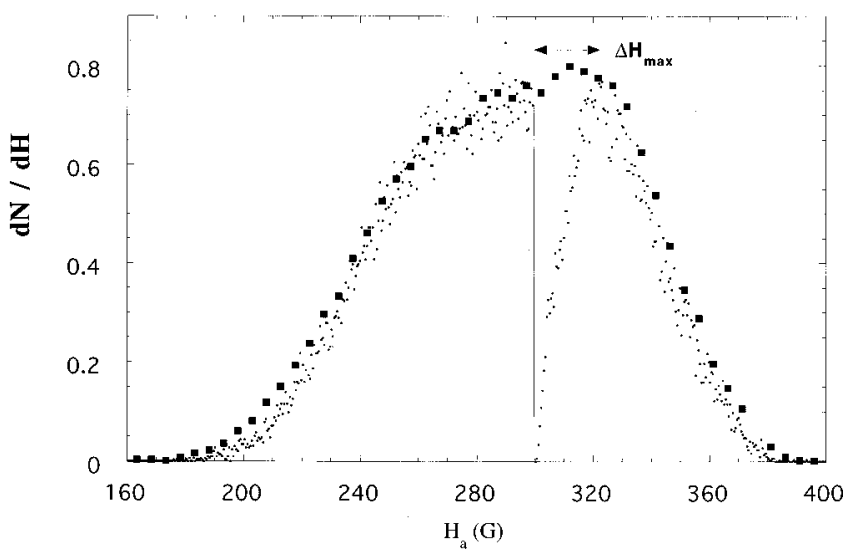

FIG. 3. Superposition of differential superheating curves $(d N / d H)$ at $480 \mathrm{mK}$, with $(\bullet)$ and without irradiation $(\mathbf{\square})$.

formity of grain metallurgy and size, it seems impossible to avoid the existence of some distribution of local magneticfield states within a phase diagram of the ensemble: the suspension constitutes homogeneous, disordered media. This spread of the local-field maxima at the grain equators can be represented schematically by the vertical line $x y$ in the phase diagram of Fig. 4, which in principle incorporates any defect presence: there is one and only one phase line for the material, given by $H_{\mathrm{sh}}(T)$. In this diagram, for a given $H_{a}$, some fraction of the suspension has transited to the normal state; each grain of the remainder sees a different maximum local field $\left(H_{l}\right)$, resulting in a distribution $\Delta T_{l}=\left[T_{\mathrm{sh}}\left(H_{l}\right)-T_{a}\right]$ required for normal-state transitions, where $T_{\mathrm{sh}}$ is the superheated transition temperature.

Irradiating the suspension results in energy loss of the incident radiation to the material, generating temperature increases in accordance with Eq. (1): in the simplest description, with sufficiently long irradiation times or intensity, all grains within the region $\Delta H_{\max }=H_{\mathrm{sh}}\left(T_{a}\right)-H_{\rho}$ of Fig. 4 , corresponding to $\Delta E_{\max }$ of Eq. (1), undergo a phase transition. The field $H_{\rho}$ corresponds to the $T_{a}+\Delta T$ of Eq. (3) for which

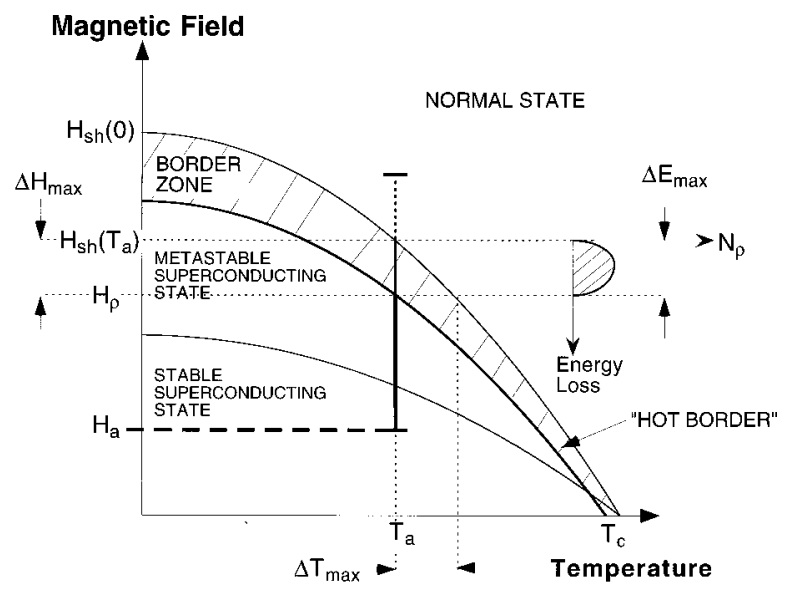

FIG. 4. An effective phase diagram of a grain's suspension. As indicated, electrons from the lower part of the energy-loss spectrum are capable of flipping only grains with small $\Delta T$. Sufficiently long irradiation times or intense radiation fields establish an effective hot border at $H_{\rho}$; grains introduced into the region between the superheated phase boundary and the "hot border" via increase of the applied magnetic field can transition only by thermal nucleation. 
Eq. (5) is satisfied. This is observed in Fig. 3: the gap in the differential superheating curve of the suspension under irradiation results from the transiting of grains to the normal state during a pause at $H_{a}=300 \mathrm{G}$ inserted into the field ramping, which has been recorded separately. The slope of the high-field region of the gap results from the transition of grains introduced into the hot border zone by increasing $H_{a}$ following the pause.

The return of the suspension's superheating curve to its unirradiated behavior occurs when $H_{\rho}$ has been raised to $H_{\mathrm{sh}}\left(T_{a}\right)$. The maximum gap width $\left(\Delta H_{\max }\right)$ thus provides a measure of $\Delta T_{\max }$ induced by the maximum energy loss of the irradiation, which is defined by the minimum $V_{\text {heated }}$ of the suspension at $H_{\rho}$. Despite the many-body complications, the energy loss remains proportional to the electron range, and $\Delta E_{\max } / V_{\text {grain }}$ varies as $R_{\min }^{-2}$. In a global-heating description, $\Delta H_{\max } \Leftrightarrow \Delta H=H_{\mathrm{sh}}-H_{\text {step }}$ of Ref. 4, $V_{\min }$ corresponds to the smallest grain of the suspension and $\Delta E_{\max } / V_{\min }=$ const for all temperature. For local-heating models, $\Delta H_{\max } \gg \Delta H$ : $V_{\min }$ continues to correspond to that of a defect, so that $\Delta H_{\max }$ scales with $V(\xi)$ and must therefore exhibit a temperature dependence.

\section{EXPERIMENT}

Measurements of $\Delta H_{\max }$ were conducted for two different suspensions of Sn grains, of 10-25 $\mu \mathrm{m}$ diameters for $T_{a}$ $>2 \mathrm{~K}$ and 16-25 $\mu \mathrm{m}$ diameters for $T_{a}<1 \mathrm{~K}$. In each case, data acquisition was accomplished using fast-pulse electronics. ${ }^{16}$ The normal-state nucleation of a grain creates discontinuities in the flux cut by a detecting loop, which is connected via two transformers to a LeCroy HQV810-based preamplifier. Only irreversible flux entry is detected: reversible flux changes are outside of the bandwidth of the preamplifier. The input signal is shaped with a fast LeCroy amplifier and discriminated with a LeCroy MVL407 ultrafast voltage comparator. Computer control synchronizes the magnetic step rise with the opening of a gate during which flux pulses are detected. Owing to the transformer presence, the current timing resolution of the system is limited to about $10 \mu \mathrm{s}$.

\section{A. Temperatures $>2 \mathrm{~K}$}

The grains were of spherical geometry, varying in diameter from $10-25 \mu \mathrm{m}$, and were part of a sample made by sonic dispersion of the molten metal in an oil bath, size filtration using calibrated sieves, and uniform folding ${ }^{17}$ into a paraffin dielectric. The suspension consisted of three disks, each $5 \mathrm{~mm}$ in diameter and $0.3 \mathrm{~mm}$ thick. Each disk was estimated to contain $1.4 \times 10^{5}$ tin grains based on a volume filling factor of $20 \%$. The disks were spaced along a $100-$ $\mu \mathrm{m}$-diameter U-shaped copper wire loop of $225 \mu \mathrm{m}$ width imprinted on an epoxy board.

Each disk was covered with one equal-diameter, tissue paper foil containing $5 \mu \mathrm{Ci}$ of evaporated ${ }^{35} \mathrm{~S}$, a pure betadecay source with an end-point energy of $167 \mathrm{keV}$, and a half-life of 82 days. Owing to the width of the sense coil, only about $7 \%$ of the total grains were sensitive to measurement.

The sensor was operated in a pumped ${ }^{4} \mathrm{He}$ refrigerator; the temperature was pressure regulated, providing a nominal
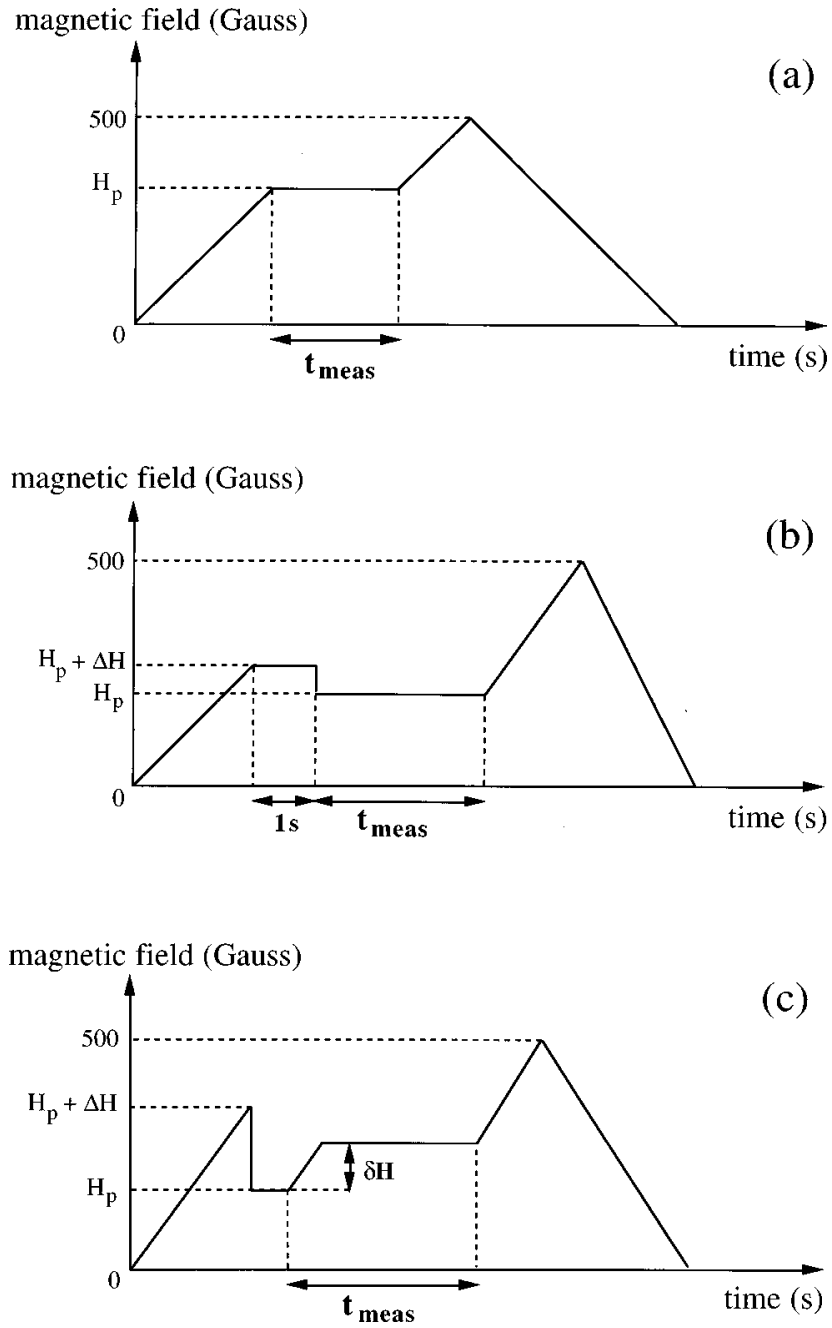

FIG. 5. Protocols of magnetic-field variation in the various experiments: (a) $T>2 \mathrm{~K}$, (b) $T<1 \mathrm{~K}$, (c) systematic test for $T$ $<1 \mathrm{~K}$, which was used to establish the time delay in (b).

stability of $10^{-3} \mathrm{~K}$. The applied magnetic field, generated perpendicular to the loop by a niobium Helmholtz coil of 10 $\mathrm{cm}$ diameter and $1 \mathrm{~cm}$ separation, provided a field stability of $0.01 \mathrm{G}$ and homogeneity of $3 \%$ over the detector region.

Measurements ${ }^{18}$ were conducted at 2.30, 3.10, and $3.44 \mathrm{~K}$ with the radiation source installed. For each measurement, the detector was tuned by first cooling the suspension at zero field. The applied field was then raised until the last grain has transited to the normal state; the threshold level of the discriminator was adjusted to the minimum level at which no noise effect was observed in the curve.

Following electronic tuning, the applied field was then ramped in increments of $0.29 \mathrm{G}$ in time intervals of $0.02 \mathrm{~s}$ at $2.30 \mathrm{~K}, 0.04 \mathrm{~s}$ at $3.10 \mathrm{~K}$, and $0.09 \mathrm{~s}$ at $3.44 \mathrm{~K}$ to a mean value in the linear part of the superheating curve (well above the region in which intermediate states may exist) and a 3-s "pause" effected before the ramping was again continued until the last grain had transited to the normal state. This protocol, shown schematically in Fig. 5(a), was followed by a return of the field to zero; it was repeated many times.

\section{B. Temperatures $<\mathbf{1} \mathrm{K}$}

Sn grains of 16-25 $\mu \mathrm{m}$ diameter were also part of a sample made by sonic dispersion of the molten metal in an 


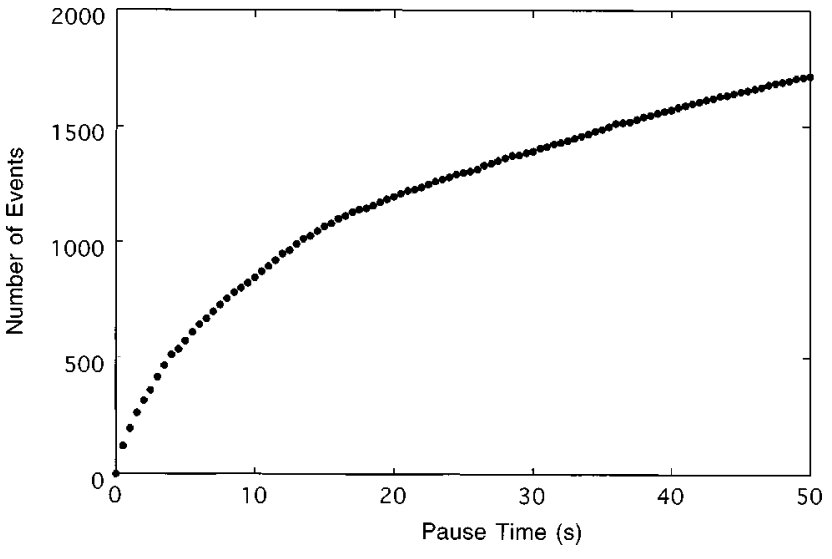

FIG. 6. A typical rate curve of Sn grains transitions induced by irradiation, obtained during a pause period at $T_{a}=850 \mathrm{mK}$.

oil bath, size filtration using calibrated sieves; they were uniformly suspended ${ }^{17}$ in paraffin to a $20 \%$ volume filling factor. The source activity, $144 \mathrm{kBq}$ of ${ }^{14} \mathrm{C}$, a pure beta decay with an end-point energy of $157 \mathrm{keV}$ and half-life of 5730 yr, was evaporated onto a thin absorbent paper. The suspension, $2.5 \times 1.5 \times 0.02 \mathrm{~cm}^{3}$, was installed on the mixing chamber of a ${ }^{3} \mathrm{He}-{ }^{4} \mathrm{He}$ dilution refrigerator without $1 \mathrm{~K}$ pot (DILUETTE), sandwiched between a U-shaped copper pickup loop and the source paper. The applied magnetic field was effected by a Helmholtz coil mounted on the barrel of the vacuum shield orthogonal to the refrigerator axis, and provided a field stability of $2 \times 10^{-2} \mathrm{G}$ with homogeneity of $1 \%$.

Measurements were performed at 100, 200, 480, and 850 $\mathrm{mK}$. The measurement protocol used in these tests was essentially that of previous researchers, ${ }^{3}$ rather than that of the $T>2 \mathrm{~K}$ tests: the applied field was first raised to $300 \mathrm{G}$ $+\Delta H$ in steps of $18 \mathrm{G} \mathrm{s}^{-1}$, then lowered to $300 \mathrm{G}$ for measurement, as shown in Fig. 5(b). This measurement field is well above the region in which intermediate states may exist. Grains that undergo a magnetically induced phase transition between $300 \mathrm{G}$ and $300 \mathrm{G}+\Delta H$ remain normal after decreasing the field to $300 \mathrm{G}$ because the phase transition is irreversible. With this protocol, a zone of depth $\Delta H$ from the superheating boundary is magnetically depopulated, and the parameter $\Delta H$ can be considered as an energy threshold. During the measurement time $\left(t_{\text {meas }}\right)$, detected transitions are only due to irradiation. The measurement period, $50 \mathrm{~s}$, was selected on the basis of the observed transition rate of the superconducting grains, which as seen in Fig. 6 is typically exponential.

Following measurement, the field was raised until all grains were in the normal state, then recycled to zero and the procedure repeated many times. The variation in $\Delta H$ was 1 G.

The pause at $H_{p}+\Delta H$ resulted from systematic tests without irradiation in which the field was raised by an amount $\delta H$ just after reaching $300 \mathrm{G}$ and before the measurement, as shown in Fig. 5(c). In this case, for $\delta H<\Delta H$, no transitions were recorded; for $\delta H>\Delta H$, transitions occurred because of the delay in the magnetic-field response as a result of the field coil damping. Because of this damping, an additional 1 -s delay at $300 \mathrm{G}+\Delta H$ was required in order for the field to reach its command value.

\section{RESULTS AND DISCUSSION}

The maximum magnetic gap widths measured in each temperature range are presented in column 2 of Table I, together with the corresponding $\Delta T_{\max }$ in column 3 . For the $T>2 \mathrm{~K}$ measurements, $\left\{\Delta T_{\max }\right\}$ was obtained via Eq. (3) with $H_{\text {sh }}^{\text {local }}(0)=570 \mathrm{G} .{ }^{6}$ The $T<1 \mathrm{~K}$ measurements, however, required a different treatment since Eq. (4) is not well established for $T_{a} \ll T_{c}$. Moreover, because of the slow variation of $H_{\mathrm{sh}}$ with temperature for this region, a small error in the determination of $H_{\mathrm{sh}}(T)$ induces a large error in $\Delta T$. In this case, $\left\{\Delta T_{\max }\right\}$ was determined from the $\Delta H$ measurement and from a separately measured $H_{\mathrm{sh}}(T)$ curve.

The last column of Table I is calculated from the integral of Eq. (1) with the $\left\{\Delta T_{\max }\right\}$ column entries using Eq. (2) with $\{a, b, \alpha, \gamma\}=\left\{7.85,1.42,0.101 \mathrm{keV} / \mathrm{K}^{4} \mu \mathrm{m}^{3}, 0.6718 \mathrm{keV} / \mathrm{K}^{2}\right.$ $\left.\mu \mathrm{m}^{3}\right\}$ for $\mathrm{Sn} .{ }^{19}$ As evident, the integral is constant within errors for each suspension over its respective temperature range.

\section{A. Variation of $\Delta E_{\max } / V$ with temperature}

The last column of Table I indicates no variation in $\Delta E_{\max } / V$ for either temperature range, especially for $T$ $>2 \mathrm{~K}$, where the effects of a $V_{\text {heated }}(\xi)$ would be expected to appear. We show in Fig. 7 these results together with the

TABLE I. Experimental maximum gap widths and associated parameters.

\begin{tabular}{lccc}
\hline \hline$T(\mathrm{~K})$ & $\Delta H_{\max }(\mathrm{G})$ & $\Delta T_{\max }(\mathrm{K})$ & $\int_{T_{a}}^{T_{a}+\Delta T_{\max }} C_{s} d T\left(\mathrm{keV} / \mu \mathrm{m}^{3}\right)$ \\
\hline$T<1 \mathrm{~K}\left(16-25-\mu \mathrm{m} \varnothing\right.$ suspension, ${ }^{14} \mathrm{C}$ irradiation $)$ & & \\
$0.85 \pm 0.04$ & $10.0 \pm 0.12$ & $0.18 \pm 0.10$ & $0.027 \pm 0.008$ \\
$0.48 \pm 0.02$ & $14.0 \pm 0.12$ & $0.51 \pm 0.08$ & $0.034 \pm 0.007$ \\
$0.20 \pm 0.02$ & $16.0 \pm 0.12$ & $0.75 \pm 0.08$ & $0.029 \pm 0.005$ \\
$0.10 \pm 0.01$ & $0.86 \pm 0.08$ & $0.030 \pm 0.007$ \\
& $17.0 \pm 0.12$ & & Mean $0.030 \pm 0.013$ \\
$T>2 \mathrm{~K}\left(10-25-\mu \mathrm{m} \varnothing\right.$ suspension, ${ }^{35} \mathrm{~S}$ irradiation) & & \\
$3.44 \pm 0.04$ & $3.2 \pm 0.1$ & $1.02 \pm 0.12$ & $0.083 \pm 0.004$ \\
$3.10 \pm 0.02$ & $3.6 \pm 0.1$ & $1.24 \pm 0.17$ & $0.081 \pm 0.005$ \\
$2.30 \pm 0.02$ & $6.3 \pm 0.1$ & $2.93 \pm 0.23$ & $0.093 \pm 0.003$ \\
& & Mean $0.086 \pm 0.004$ \\
\hline \hline
\end{tabular}




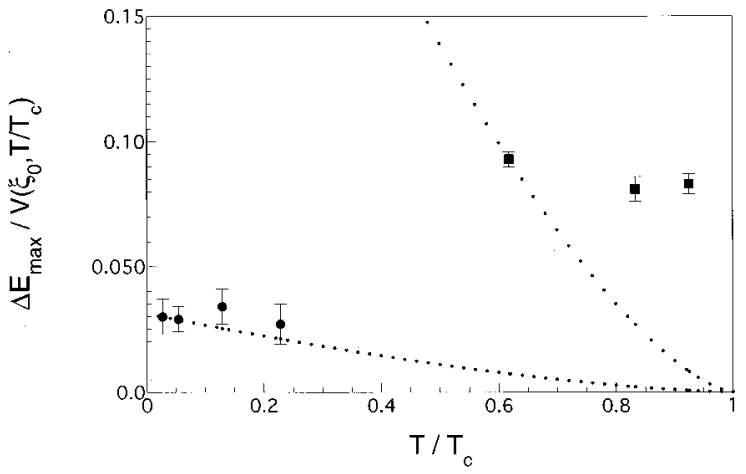

FIG. 7. Comparison of the experimental $\Delta E_{\max } / V_{\text {heated }}$ with the predicted temperature dependence of the coherence length; $V(\xi)$ has been normalized to reproduce the lowest temperature result in each temperature range.

temperature variation of $\Delta E_{\max } / V(\xi)$ anticipated from the lowest temperature result in each temperature range. For $T$ $<1 \mathrm{~K}$, where $\xi \approx \xi_{0}$, the results are insufficiently sensitive to be conclusive; however, the normalization implies $R_{\text {heated }}$ $\approx 35 \xi_{0}$ suggesting a $V_{\text {heated }}$ much larger than would be expected by any local-heating description. For the $T>2 \mathrm{~K}$ results, the sensitivity is significantly better than the predicted temperature variation.
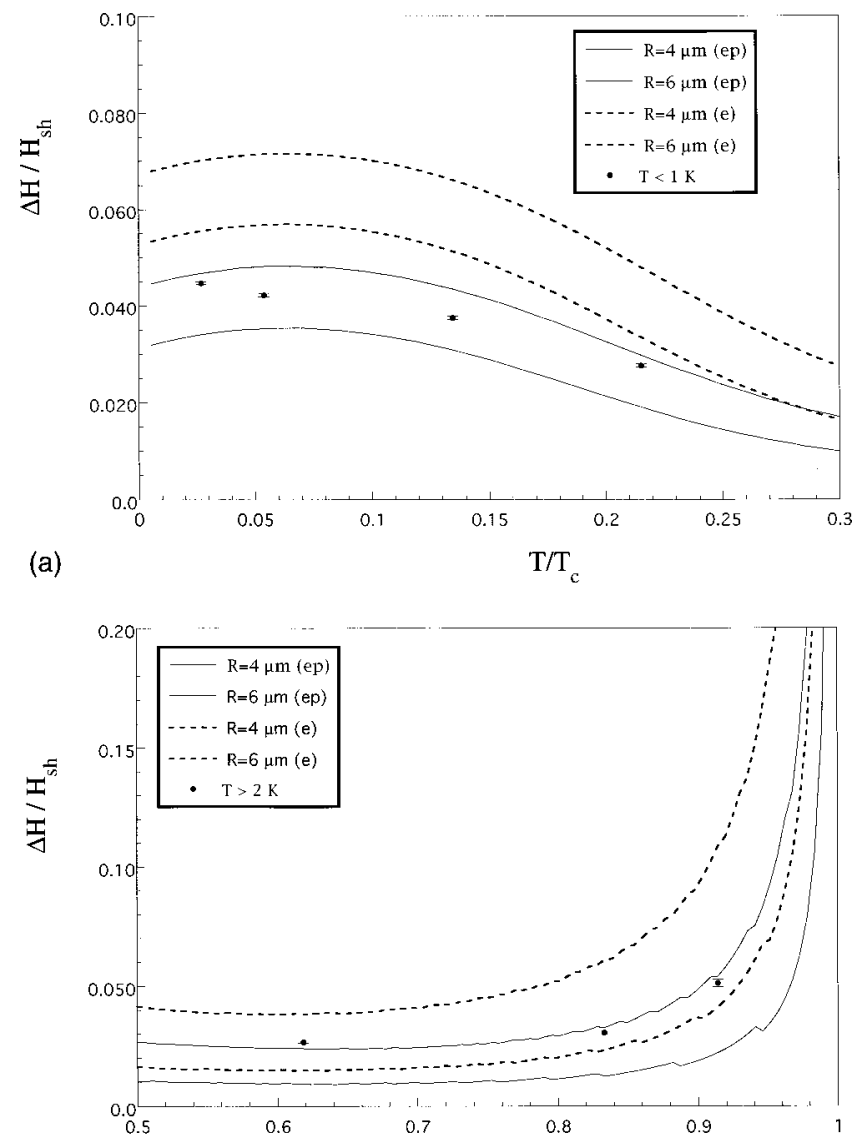

(b)

$\mathrm{T} / \mathrm{T}_{\mathrm{c}}$

FIG. 8. Calculation of the maximum field gaps as a function of temperature, with (-) and without (---) the phonon contribution to the specific heat: (a) $T<1 \mathrm{~K}$, (b) $T>2 \mathrm{~K}$.

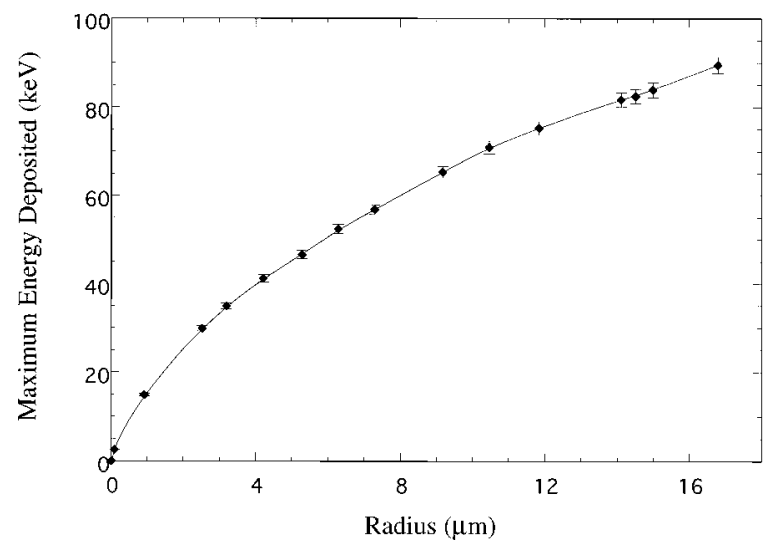

FIG. 9. The results of simulation of the maximum electron energy loss from the decay of ${ }^{14} \mathrm{C}$ in $\mathrm{Sn}$ microspheres of varying diameter, as described in the text.

\section{B. Heating of the phonon system}

Analysis of the phonon system warming, corresponding to Figs. 4 and 5 of Ref. 4, is shown in Fig. 8, together with the measured $\left\{\Delta H_{\max }\right\}$ from Table I. The contours are obtained by computing the $T+\Delta T_{\max }$ in the integral of Eq. (1), which is necessary to provide the same value of $\Delta E_{\max } / V_{\min }$ to within $1 \%$. The area defined within the solid lines represents the results for $C_{s}=C_{\text {electron }}+C_{\text {phonon }}$, with $R_{\text {min }}$ $=R_{\text {measurement }} \pm 1 \mu \mathrm{m}$; the area within the dotted lines, $C_{s}$ $=C_{\text {electron }}$ only. For the $T>2 \mathrm{~K}$ data, the theoretical contours overlap and the data supports either hypothesis; the $T<1 \mathrm{~K}$ results, however, lie well within the area anticipated from a full specific heat (electron + phonon), in contrast to the corresponding Fig. 5 of Ref. 4: nucleation of the normal state proceeds with a fully warmed phonon system.

\section{Heated volumes}

The $\Delta E_{\max }$ of each decay for different grain radii was computed using a simulation ${ }^{20}$ that randomly emits electrons from a distributed source consistent with the energy distribution of the decay, and tracks them through both the paraffin and grains in accordance with Moliere scattering; ${ }^{21}$ the energy loss in each medium is computed using the Bethe-Bloch equation. ${ }^{22}$ Figure 9 shows the results for the ${ }^{14} \mathrm{C}$ decay electrons. For the minimum radius of the $T>2 \mathrm{~K}$ and $T<1 \mathrm{~K}$ ensembles, $\Delta E_{\max }=45 \pm 1$ and $65 \pm 1 \mathrm{keV}$, respectively. Divided by the respective mean values of the last column of Table I, these yield $R_{\text {heated }}$ of $8.0 \pm 2.1$ and $5.0 \pm 0.086 \mu \mathrm{m}$ for the $T<1 \mathrm{~K}$ and $T>2 \mathrm{~K}$ measurements, respectively, equivalent to the smallest grain radius in each suspension and well above $\xi_{0}$.

According to the discussion of Sec. II A, the local-heating behavior observed in Ref. 4 for $\mathrm{Sn}$ and In is characterized by a low-energy tail in the differential superheating curves extending $30-50 \mathrm{G}$ below $H_{\text {step }}$ (see Fig. 3 therein); in principle, the lowest recorded transition field of this tail should correspond to the nearest-defect energy deposition, and $V_{\text {heated }} \approx V(\xi)$. In fact, these lowest transition fields imply $R_{\text {heated }} \approx 8.5 \mu \mathrm{m}$, well above $\xi_{0}$ but sufficiently less than $R_{\text {grain }}$ to further suggest the intermediate-state presence. 


\section{CONCLUSIONS}

In order to investigate the local-heating conclusion of Ref. 4 for $\mathrm{Sn}$, we have performed a series of electron irradiation experiments on two different, fully metastable, suspensions of $\mathrm{Sn}$ grains in two different temperature ranges below $T_{c}$.

Measurements for $T<1 \mathrm{~K}$ with a suspension of $\mathrm{Sn}$ grains $16-25 \mu \mathrm{m}$ in diameter irradiated by the decay electrons of ${ }^{14} \mathrm{C}$ clearly demonstrate that nucleation of the normal state proceeds with a fully warmed phonon system; measurements for $T>2 \mathrm{~K}$ with a suspension of $\mathrm{Sn}$ grains $10-25 \mu \mathrm{m}$ in diameter irradiated by ${ }^{35} \mathrm{~S}$ decay electrons clearly deny both the temperature variation of $\Delta E_{\max } / V(\xi)$ and $V_{\text {heated }}$ $\approx 0.5 V_{\text {min }}$. In each case, the heated volume derived from the maximum gap width induced in the respective superheating curves by the irradiation, combined with the calculated maximum energy deposition in the material, is in good agreement with the smallest grain of its suspension.

We thus conclude that the electron-induced thermal nucleation of a suspension of superheated superconducting $\mathrm{Sn}$ grains is consistent with a global heating of the grain volume, contrary to the model of Ref. 4.

The energy of the $\alpha$ irradiation of Ref. 4 experiments is generally deposited within $10 \mu \mathrm{m}$ of the grain surface. Although the electrons of these experiments have a maximum range of some $70 \mu \mathrm{m}$ in $\mathrm{Sn}$, about $40 \%$ of the incident activity is also stopped within $10 \mu \mathrm{m}$, with energy sufficient to raise a defect volume by several $\mathrm{K}$ throughout the measurement range. However, the distinction between local- and global-heating behavior of the Ref. 4 model is based on whether or not the material-dependent quasiparticle relaxation rate is less than the diffusion rate, independent of the energy deposition mechanism. The defect presence is sig- naled by the step at $H_{\text {step }}$ in the measurements of Ref. 4, but the local-heating behavior is manifested by the low-field tail below $H_{\text {step }}$. According to Ref. 4, the presence of this tail in $\mathrm{Sn}$ is explained by the more rapid quasiparticle relaxation rather than diffusion rate. This tail is also observed in the In irradiations, and a local-heating behavior is similarly concluded for the same reason; for $\mathrm{Al}$ and $\mathrm{Zn}$, no tail is observed and these transitions are designated as "global" because the relaxation rate is slower than the diffusion. Observation of the local-heating tail in the $\mathrm{Sn} / \mathrm{In}$ experiments of Ref. 4 is, however, most likely due only to the significant presence of intermediate states, evidenced by the fact that the grains never reached the theoretical limit of superheating as seen in the experimental superheating fields. Since this can be determined in advance by measurement of the associated differential superheating curve, we strongly recommend that this curve be examined prior to the future reporting of any such experiments.

Curiously, the model of Ref. 4 would seem to "make sense" physically. Since it constitutes a reasonable first attempt at explaining the heat transport in a type-I superconducting material, why it fails to manifest itself in our experiments is perhaps a greater question. Further experimental investigations are clearly needed.

\section{ACKNOWLEDGMENTS}

This work was supported in part by JNICT Grants No. C/FAE/1051/95 and No. C/FAE/1110/96 under Programa CERN of Portugal. Two of us (T.A.G. and J.I.C.) again thank the CNRS and Groupe de Physique des Solides of Universités Paris 7/6 for their hospitality and support during this work.
${ }^{1}$ G. Waysand, in Superconductivity and Particle Detection, edited by T. A. Girard, A. Morales, and G. Waysand (World Scientific, Singapore, 1995), p. 1.

${ }^{2}$ J. Feder and D. McLachlin, Phys. Rev. 177, 763 (1969).

${ }^{3}$ M. Frank, P. Freund, J. Gebauer, K. Pretzl, A. Singsaas, and L. Stodolsky, Nucl. Instrum. Methods Phys. Res. A 287, 583 (1990); J. Fent, P. Freund, G. Gebauer, K. Pretzl, N. Schmitz, A. Singsaas, L. Stodolsky, and G. Vesztergombi, in Low Temperature Detectors for Neutrinos and Dark Matter, edited by K. Pretzl, N. Schmitz, and L. Stodolsky (Springer-Verlag, Berlin, 1987), p. 30.

${ }^{4}$ M. Frank, A. Singsaas, L. Stodolsky, and S. Cooper, Phys. Rev. B 43, 5321 (1991).

${ }^{5}$ S. B. Kaplan, C. C. Chi, D. N. Langenberg, J. J. Chang, S. Jafarey, and D. J. Scalapino, Phys. Rev. B 14, 4854 (1976).

${ }^{6}$ A. Larrea, A. Morales, G. Waysand, and J. Bartolome, Nucl. Instrum. Methods Phys. Res. A 317, 541 (1992).

${ }^{7}$ F. W. Smith, A. Baratoff, and M. Cardona, Phys. Kondens. Mater. 12, 145 (1979).

${ }^{8}$ D. K. Finnemore and D. E. Mapother, Phys. Rev. 140, A507 (1965).

${ }^{9}$ P. G. de Gennes, Superconductivity of Metals and Alloys (Addison-Wesley, New York, 1966), p. 40.

${ }^{10}$ E. V. Thuneberg, J. Low Temp. Phys. 62, 27 (1986).
${ }^{11}$ M. Tinkham, Introduction to Superconductivity (McGraw-Hill, New York, 1996), p. 119.

${ }^{12}$ D. Hueber, C. Valette, and G. Waysand, Physica B \& C 108, 1229 (1981).

${ }^{13}$ This expression is customarily stated as valid only for $T \approx T_{c}$; a more general validity over the phase space near the transition boundary follows from the work of Gork'ov and others. Moreover, high-field calculations of $H_{c 2}$ (treated in, for example, Refs. 9 or 11) yield $H_{c 2} \approx \xi^{-2}(t)$; measurements of this field over $0<t<1$ (for example, Refs. 2, 6, and 7) yield $H_{c 3} / H_{c}$ $\approx(1-t)^{1 / 6} \rightarrow \xi(t) \approx(1-t)^{-1 / 2}$.

${ }^{14} \mathrm{U}$. Geigenmueller, in Superconducting and Low-Temperature Particle Detectors, edited by G. Waysand and G. Chardin (Elsevier, Amsterdam, 1989), p. 191; J. Phys. (France) 49, 405 (1988).

${ }^{15}$ Several groups have reduced this spreading an order of magnitude by resorting to ordered arrays [for microspheres, see, for example, M. Le Gros, A. da Silva, B. G. Turrell, A. Kotlicki, and A. K. Drukier, Appl. Phys. Lett. 56, 2234 (1990); for microdots, see, for example, R. Leoni, G. Shirripa Spagnola, P. Carelli, M. G. Castellano, and M. Cirillo, in Low Temperature Detectors for Neutrinos and Dark Matter, edited by N. Booth and G. L. Salmon (Editions Frontieres, Paris, 1992), p. 57]. None have, however, been able to eliminate it. 
${ }^{16}$ A. de Bellefon, D. Broszkiewicz, R. Bruère-Dawson, P. Espigat, and D. Sotiras, Nucl. Instrum. Methods Phys. Res. A 277, 211 (1989).

${ }^{17}$ V. Jeudy, D. Limagne, and G. Waysand, Nucl. Phys. B, Proc. Suppl. 28A, 482 (1992).

${ }^{18}$ T. A. Girard, R. P. Henriques, J. I. Collar, M. Godinho, G. Bonfait, G. Waysand, D. Limagne, V. Jeudy, and V. Pagesy, Nucl. Instrum. Methods Phys. Res. A 334, 645 (1993).
${ }^{19}$ H. R. O’Neal and N. E. Phillips, Phys. Rev. 137, A748 (1965).

${ }^{20}$ J. I. Collar, T. A. Girard, and G. Waysand, J. Low Temp. Phys. 93, 497 (1993).

${ }^{21}$ G. R. Lynch and O. I. Dahl, Nucl. Instrum. Methods Phys. Res. B 58, 6 (1991); Phys. Lett. B 239, 13 (1990).

${ }^{22}$ R. M. Sternheimer, M. J. Berger, and S. M. Seltzer, At. Data Nucl. Data Tables 30, 261 (1984). 